PROCEEDINGS OF THE

AMERICAN MATHEMATICAL SOCIETY

Volume 136, Number 9, September 2008, Pages 3211-3221

S 0002-9939(08)09300-3

Article electronically published on April 30, 2008

\title{
THE LAPLACE TRANSFORM OF THE DIGAMMA FUNCTION: AN INTEGRAL DUE TO GLASSER, MANNA AND OLOA
}

\author{
TEWODROS AMDEBERHAN, OLIVIER ESPINOSA, AND VICTOR H. MOLL
}

(Communicated by Carmen C. Chicone)

Abstract. The definite integral

$$
M(a):=\frac{4}{\pi} \int_{0}^{\pi / 2} \frac{x^{2} d x}{x^{2}+\ln ^{2}\left(2 e^{-a} \cos x\right)}
$$

is related to the Laplace transform of the digamma function

$$
L(a):=\int_{0}^{\infty} e^{-a s} \psi(s+1) d s,
$$

by $M(a)=L(a)+\gamma / a$ when $a>\ln 2$. Certain analytic expressions for $M(a)$ in the complementary range, $0<a \leq \ln 2$, are also provided.

\section{INTRODUCTION}

The classical table of integrals by I. S. Gradshteyn and I. M. Ryzhik [7] contains a large collection organized in sections according to the form of the integrand. In each section one finds a significant variation on the complexity of the integrals. For example, section 4.33-4.34, with the title Combinations of logarithms and exponentials, presents the elementary formula 4.331.1. For $a>0$,

$$
\int_{0}^{\infty} e^{-a x} \ln x d x=-\frac{\gamma+\ln a}{a},
$$

where $\gamma$ is the Euler constant

$$
\gamma=\lim _{n \rightarrow \infty} \sum_{k=1}^{n} \frac{1}{k}-\ln n
$$

Contained in the same section are the more elaborate 4.332.1 and 4.325.6:

$$
\int_{0}^{\infty} \frac{\ln x d x}{e^{x}+e^{-x}-1}=\int_{0}^{1} \ln \ln \left(\frac{1}{x}\right) \frac{d x}{x^{2}-x+1}=\frac{2 \pi}{\sqrt{3}}\left(\frac{5}{6} \ln 2 \pi-\ln \Gamma\left(\frac{1}{6}\right)\right) .
$$

The difficulty involved in the evaluation of a definite integral is hard to measure from the complexity of the integrand. For instance, the evaluation of Vardi's integral,

$$
\int_{\pi / 4}^{\pi / 2} \ln \ln \tan x d x=\int_{0}^{1} \ln \ln \left(\frac{1}{x}\right) \frac{d x}{1+x^{2}}=\frac{\pi}{2} \ln \left(\frac{\Gamma\left(\frac{3}{4}\right) \sqrt{2 \pi}}{\Gamma\left(\frac{1}{4}\right)}\right),
$$

Received by the editors July 23, 2007.

2000 Mathematics Subject Classification. Primary 33B15.

Key words and phrases. Laplace transform, digamma function.

The work of the third author was partially funded by NSF-DMS 0409968.

(C)2008 American Mathematical Society 
that appears as 4.229.7 in [7, requires a reasonable amount of number theory. The second integral form is 4.325.4, found in the section entitled Combinations of logarithmic functions of more complicated arguments and powers. The reader will find in [15] a discussion of this formula.

It is a remarkable fact that combinations of elementary functions in the integrand often exhibit definite integrals whose evaluation is far from elementary. A systematic study of the formulas in [7] has been initiated in the series [1, 2, 9, 10, 11, 12, These papers are organized according to the combinations appearing in the integrand. Even the elementary cases, such as the combination of logarithms and rational functions discussed in [2], entail interesting results. The evaluation

$$
\begin{aligned}
\int_{0}^{b} \frac{\ln t d t}{(1+t)^{n+1}} & =\frac{1}{n}\left[1-(1+b)^{-n}\right] \ln b-\frac{1}{n} \ln (1+b) \\
& -\frac{1}{n(1+b)^{n-1}} \sum_{j=1}^{n-1} \frac{1}{j !}\left(\begin{array}{c}
n-1 \\
j
\end{array}\right)|s(j+1,2)| b^{j}
\end{aligned}
$$

for $b>0$ and $n \in \mathbb{N}$, produces an explicit formula for the case where the rational function has a single pole. Here, $|s(n, k)|$ are the unsigned Stirling numbers of the first kind, which count the number of permutations of $n$ letters having exactly $k$ cycles. The case of a purely imaginary pole,

$$
\int_{0}^{x} \frac{\ln t d t}{\left(1+t^{2}\right)^{n+1}}=\frac{\left(\begin{array}{c}
2 n \\
n
\end{array}\right)}{2^{2 n}}\left[g_{0}(x)+p_{n}(x) \ln x-\sum_{k=0}^{n-1} \frac{\tan ^{-1} x+p_{k}(x)}{2 k+1}\right],
$$

is expressed in terms of the rational function

$$
p_{n}(x)=\sum_{j=1}^{n} \frac{2^{2 j}}{2 j\left(\begin{array}{c}
2 j \\
j
\end{array}\right)} \frac{x}{\left(1+x^{2}\right)^{j}},
$$

and with

$$
g_{0}(x)=\ln x \tan ^{-1} x-\int_{0}^{x} \frac{\tan ^{-1} t}{t} d t .
$$

The special case $x=1$ becomes

$$
\int_{0}^{1} \frac{\ln t d t}{\left(1+t^{2}\right)^{n+1}}=-2^{-2 n}\left(\begin{array}{c}
2 n \\
n
\end{array}\right)\left(G+\sum_{k=0}^{n-1} \frac{\frac{\pi}{4}+p_{k}(1)}{2 k+1}\right),
$$

where

$$
G=\sum_{k=0}^{\infty} \frac{(-1)^{k}}{(2 k+1)^{2}}
$$

is Catalan's constant. The values

$$
p_{k}(1)=\sum_{j=1}^{k} \frac{2^{j}}{2 j\left(\begin{array}{c}
2 j \\
j
\end{array}\right)}
$$

do not admit a closed form (in the sense of [14]), but they do satisfy the three-term recurrence

$$
(2 k+1) p_{k+1}(1)-(3 k+1) p_{k}(1)+k p_{k-1}(1)=0
$$


The study of definite integrals, where the integrand is a combination of powers, logarithms and trigonometric functions, was initiated by Euler [5], with the evaluation of

$$
\int_{0}^{\pi / 2} x \ln (2 \cos x) d x=-\frac{7}{16} \zeta(3)
$$

and

$$
\int_{0}^{\pi / 2} x^{2} \ln (2 \cos x) d x=-\frac{\pi}{4} \zeta(3)
$$

which appear in his study of the Riemann zeta function at the odd integers. These type of integrals have been investigated in [8], [16]. The intriguing integral of D. and J. Borwein [3],

$$
\int_{0}^{\pi / 2} x^{2} \ln ^{2}(2 \cos x) d x=\frac{11 \pi}{16} \zeta(4)=\frac{11 \pi^{5}}{1440},
$$

was first conjectured on the basis of a numerical computation by Enrico Au-Yueng while he was an undergraduate student at the University of Waterloo. A nice example of experimental mathematics in action.

Recently O. Oloa considered the integral

$$
M(a):=\frac{4}{\pi} \int_{0}^{\pi / 2} \frac{x^{2} d x}{x^{2}+\ln ^{2}\left(2 e^{-a} \cos x\right)},
$$

and the special value

$$
M(0)=\frac{4}{\pi} \int_{0}^{\pi / 2} \frac{x^{2} d x}{x^{2}+\ln ^{2}(2 \cos x)}=\frac{1}{2}(1+\ln (2 \pi)-\gamma)
$$

is established in 13 .

Oloa's method of proof relies on the expansion

$$
\frac{x^{2}}{x^{2}+\ln ^{2}(2 \cos x)}=x \sin 2 x+\sum_{n=1}^{\infty}(-1)^{n-1}\left(\frac{a_{n}}{n !}-\frac{a_{n+1}}{(n+1) !}\right) x \sin (2 n x) .
$$

Here

$$
a_{n}:=\int_{0}^{1}(t)_{n} d t
$$

where $(t)_{n}=t(t+1) \cdots(t+n-1)$ is the Pochhammer symbol. The standard relation

$$
(t)_{n}=\sum_{k=1}^{n}|s(n, k)| t^{k}
$$

gives

$$
a_{n}=\sum_{k=1}^{n} \frac{|s(n, k)|}{k+1}
$$

M. L. Glasser and D. Manna [6] introduced the function

$$
L(a):=\int_{0}^{\infty} e^{-a s} \psi(s+1) d s
$$


where $\psi(x)=\frac{d}{d x} \ln \Gamma(x)$ is the digamma function. After integrating by parts and making use of (1.1), one finds

$$
L(a)=-\gamma-\ln a+a \int_{0}^{\infty} e^{-a t} \ln \Gamma(t) d t .
$$

The main result in [6] gives a relation between $M(a)$ and $L(a)$.

Theorem 1.1. If $a>\ln 2$, then

$$
M(a)=L(a)+\frac{\gamma}{a}
$$

That is, for $a>\ln 2$,

$$
M(a)=\frac{\gamma}{a}-\gamma-\ln a+a \int_{0}^{\infty} e^{-a t} \ln \Gamma(t) d t .
$$

The proof in [6] begins with the representation

$$
\int_{0}^{\pi / 2} \cos ^{\nu} x \cos a x d x=\frac{\pi \Gamma(\nu+2)}{2^{\nu+1}(\nu+1) \Gamma\left(1+\frac{\nu}{2}+\frac{a}{2}\right) \Gamma\left(1+\frac{\nu}{2}-\frac{a}{2}\right)}
$$

(3.631.9 in [7). Differentiating with respect to $a$, evaluating at $a=s$, and using $\psi(1)=-\gamma$ yields

$$
\psi(s+1)=\frac{2^{s+2}}{\pi} \int_{0}^{\pi / 2} x \cos ^{s} x \sin (s x) d x-\gamma .
$$

Replacing (1.24) into (1.20) produces

$$
L(a)+\frac{\gamma}{a}=-\frac{4}{\pi} \operatorname{Im} \int_{0}^{\infty} \int_{0}^{\pi / 2} x e^{s\left(\ln \left[2 e^{-a} \cos x\right]-i x\right)} d x d s .
$$

The identity (1.22) follows from evaluating the $s$-integral as

$$
\int_{0}^{\infty} e^{s\left(\ln \left[2 e^{-a} \cos x\right]-i x\right)} d s=\frac{1}{i x-\ln \left[2 e^{-a} \cos x\right]} .
$$

The authors of [6] produced a series expansion for $M(a)$, which they recognize as a hypergeometric function in two variables, and state that this strongly suggests that for a general value of a, no further progress is possible. The hypergeometric expression gives

$$
M(0)=1+\frac{1}{2} \int_{0}^{1} t(1-t){ }_{3} F_{2}(1,1,2-t ; 2,3 ; 1) d t
$$

on which they invoke

$$
{ }_{3} F_{2}(1,1,2-t ; 2,3 ; 1)=\frac{2(1-\gamma-\psi(t+1))}{1-t}
$$

to give a new proof of (1.15).

The graph of $M(a)$ shown in Figure 1, obtained by the numerical integration of (1.14), has a well-defined cusp at $a=\ln 2$. In this paper, analytic expressions for both branches of $M(a)$ are provided. The region $a>\ln 2$, determined in [6], has been reviewed in the present section. The corresponding expressions for $0<a<\ln 2$ is the content of the next section. 


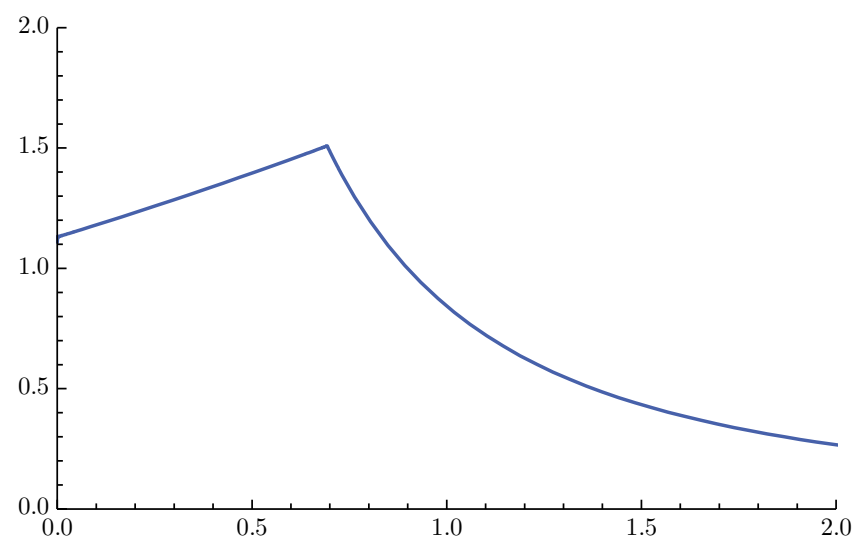

FiguRe 1. The graph of $M(a)$ for $0 \leq a \leq 2$

2. THE CASE $0<a<\ln 2$

The representation

$$
M(a)=-\frac{e^{a}}{2 \pi} \operatorname{Im} \int_{0}^{1} e^{-a t} \int_{-\pi}^{\pi} \frac{x\left(1+e^{i x}\right)^{t}}{1-e^{a}+e^{i x}} d x d t
$$

was established in [6]. Their proof is replicated here for the sake of the reader's convenience. The identity

$$
\operatorname{Im} \frac{x}{i x+\ln \left[2 e^{-a} \cos x\right]}=\frac{x^{2}}{x^{2}+\ln ^{2}\left[2 e^{-a} \cos x\right]}
$$

yields

$$
M(a)=\frac{4}{\pi} \operatorname{Im} \int_{0}^{\pi / 2} \frac{x d x}{i x+\ln \left[2 e^{-a} \cos x\right]} .
$$

If $a>\ln 2$, then

$$
\int_{0}^{\infty} e^{s \ln \left[2 e^{-a} \cos x\right]+i x} d s=\frac{1}{i x+\ln \left[2 e^{-a} \cos x\right]} .
$$

This implies

$$
M(a)=\frac{2}{\pi} \operatorname{Im} \int_{-\pi / 2}^{\pi / 2} \int_{0}^{\infty} x e^{s\left(\ln \left[2 e^{-a} \cos x\right]+i x\right)} d x d s,
$$

where one uses the fact that the imaginary part of the integrand is an even function of $x$. One more identity,

$$
e^{i s x} \cdot e^{s \ln \left[2 e^{-a} \cos x\right]}=e^{s \ln \left[e^{-a}\left(1+e^{2 i x}\right)\right]},
$$

and the change of variables $x \mapsto x / 2$, give the equality

$$
M(a)=\frac{1}{2 \pi} \operatorname{Im} \int_{-\pi}^{\pi} \int_{0}^{\infty} x e^{s \ln \left[e^{-a}\left(1+e^{i x}\right)\right]} d s d x .
$$

Evaluating the $s$-integral yields

$$
M(a)=-\frac{1}{2 \pi} \operatorname{Im} \int_{-\pi}^{\pi} \frac{x d x}{\ln \left[e^{-a}\left(1+e^{i x}\right)\right]} .
$$


The formula

$$
\frac{1}{\ln u}=\int_{0}^{1} \frac{u^{t} d t}{u-1}
$$

now gives (2.1) from (2.8).

Note 2.1. The proof outlined above is valid for $a>\ln 2$, but (2.1) holds for $a>0$.

Notation. Define $b:=e^{a}-1$ and let $0<a<\ln 2$ so that $0<b<1$.

The terms $\left(1+e^{i x}\right)^{t}$ and $1 /\left(1-b e^{-i x}\right)$ from (2.1) are now expanded in a power series to produce

$$
M(a)=-\frac{e^{a}}{2 \pi} \int_{0}^{1} \int_{-\pi}^{\pi} x e^{-a t} \sum_{j=0}^{\infty} \sum_{k=0}^{\infty} b^{j}\left(\begin{array}{l}
t \\
k
\end{array}\right) \sin [x(k-j-1)] d x d t
$$

The term corresponding to $k=j+1$ disappears, and a computation of the $x$-integral gives

$$
\begin{aligned}
M(a) & =e^{a} \int_{0}^{1} e^{-a t} \sum_{j=0}^{\infty} \sum_{k=0}^{j}(-1)^{j-k} \frac{b^{j}\left(\begin{array}{l}
t \\
k
\end{array}\right)}{j+1-k} d t \\
& +e^{a} \int_{0}^{1} e^{-a t} \sum_{j=1}^{\infty} b^{j} \sum_{\nu=1}^{j} \frac{(-1)^{\nu}}{\nu}\left(\begin{array}{c}
t \\
\nu+j
\end{array}\right) d t .
\end{aligned}
$$

Lemma 2.1. Let $t \in \mathbb{R}$ and $j \in \mathbb{N} \cup\{0\}$. Then

$$
\sum_{\nu=1}^{\infty} \frac{(-1)^{\nu}}{\nu}\left(\begin{array}{c}
t \\
\nu+j
\end{array}\right)=\left(\begin{array}{l}
t \\
j
\end{array}\right)[\psi(j+1)-\psi(t+1)] .
$$

Proof. The integral representation (3.268.2 in [7])

$$
\psi(p+1)-\psi(q+1)=-\int_{0}^{1} \frac{x^{p}-x^{q}}{1-x} d x
$$

yields

$$
\psi(p+1)-\psi(q+1)=\sum_{j=1}^{\infty}(-1)^{j-1}\left(\left(\begin{array}{l}
p \\
j
\end{array}\right)-\left(\begin{array}{l}
q \\
j
\end{array}\right)\right) .
$$

The result now follows from the identity

$$
\left(\begin{array}{l}
t \\
k
\end{array}\right)^{-1} \sum_{m=1}^{\infty} \frac{(-1)^{m}}{m}\left(\begin{array}{c}
t \\
m+k
\end{array}\right)-\sum_{m=1}^{\infty} \frac{(-1)^{m}}{m}\left(\begin{array}{c}
t \\
m
\end{array}\right)=\sum_{m=1}^{k} \frac{(-1)^{m-1}}{m}\left(\begin{array}{c}
k \\
m
\end{array}\right) .
$$

Apply the difference operator $\Delta a(k):=a(k+1)-a(k)$ and use

$$
\left(\begin{array}{c}
t \\
k+1
\end{array}\right)^{-1}\left(\begin{array}{c}
t \\
m+k+1
\end{array}\right)-\left(\begin{array}{c}
t \\
k
\end{array}\right)^{-1}\left(\begin{array}{c}
t \\
m+k
\end{array}\right)=-\frac{m}{k+1}\left(\begin{array}{c}
t \\
k+1
\end{array}\right)^{-1}\left(\begin{array}{c}
t+1 \\
m+k+1
\end{array}\right)
$$

to write the derived equation as

$$
-\frac{\left(\begin{array}{c}
t \\
k+1
\end{array}\right)^{-1}}{k+1} \sum_{m=1}^{\infty}(-1)^{m}\left(\begin{array}{c}
t+1 \\
m+k+1
\end{array}\right)=\Delta \sum_{m=1}^{k} \frac{(-1)^{m}}{m}\left(\begin{array}{c}
k \\
m
\end{array}\right) .
$$


The left hand side of (2.14) reduces to $-1 /(k+1)$ in view of the classical identity

$$
\sum_{m=1}^{\infty}(-1)^{m-1}\left(\begin{array}{c}
t+1 \\
m+k+1
\end{array}\right)=\left(\begin{array}{c}
t \\
k+1
\end{array}\right)
$$

A simple evaluation of the right hand side in (2.14) also produces $-1 /(k+1)$. Therefore both sides of (2.14) are, up to a constant, the harmonic number $H_{k}$. The special case $k=0$ shows that this constant vanishes.

Continuing from (2.10), it follows that

$$
\begin{aligned}
M(a) & =e^{a} \int_{0}^{1} \sum_{j=0}^{\infty} b^{j} \sum_{k=0}^{j} \frac{(-1)^{j-k}\left(\begin{array}{l}
t \\
k
\end{array}\right)}{j+1-k}+\frac{e^{a}}{b} \int_{0}^{1} e^{-a t} \sum_{j=1}^{\infty} b^{j}\left(\begin{array}{l}
t \\
j
\end{array}\right) \psi(j+1) d t \\
& -\frac{e^{a}}{b} \int_{0}^{1} e^{-a t} \sum_{j=1}^{\infty} b^{j}\left(\begin{array}{l}
t \\
j
\end{array}\right) \psi(t+1) d t \equiv M_{1}+M_{2}+M_{3} .
\end{aligned}
$$

To simplify $M_{1}$, observe

$$
\begin{aligned}
\sum_{j=0}^{\infty} b^{j} \sum_{k=0}^{j} \frac{(-1)^{j-k}\left(\begin{array}{l}
t \\
k
\end{array}\right)}{j+1-k} & =\sum_{k=0}^{\infty} b^{k}\left(\begin{array}{l}
t \\
k
\end{array}\right) \sum_{\nu=0}^{\infty} \frac{(-1)^{\nu} b^{\nu}}{\nu+1} \\
& =\frac{\ln (1+b)}{b} \sum_{k=0}^{\infty}\left(\begin{array}{l}
t \\
k
\end{array}\right) b^{k}=\frac{a e^{a t}}{b} .
\end{aligned}
$$

Thus, $M_{1}=a /\left(1-e^{-a}\right)$.

The reduction of $M_{2}$ employs the following result.

Lemma 2.2. If $0<a<\ln 2$, then

$$
\int_{0}^{1} e^{-a t} \sum_{j=0}^{\infty} b^{j}\left(\begin{array}{l}
t \\
j
\end{array}\right) \psi(j+1) d t=\ln \left(1-e^{-a}\right)+\int_{1}^{\infty} \frac{e^{-a t}}{t} d t
$$

Proof. The Stirling numbers $s(j, k)$ satisfy

$$
j !\left(\begin{array}{l}
t \\
j
\end{array}\right)=\sum_{k=0}^{j} s(j, k) t^{k}
$$

so that

$$
\int_{0}^{1} e^{-a t} \sum_{j=0}^{\infty} b^{j}\left(\begin{array}{l}
t \\
j
\end{array}\right) \psi(j+1) d t=\frac{e^{-a} b \gamma}{a}+e^{-a} \sum_{j=1}^{\infty}\left(b^{j+1} \alpha_{j}-b^{j} \alpha_{j-1}\right) \psi(j+1),
$$

with

$$
\alpha_{j}(a)=\frac{1}{j !} \sum_{k=0}^{j} \frac{s(j, k) k !}{a^{k+1}} .
$$

The result now follows from integration by parts and the identity

$$
\sum_{j=k}^{\infty} \frac{s(j, k) b^{j}}{j !}=\frac{\ln ^{k}(1+b)}{k !} .
$$


Therefore,

$$
M_{2}=\frac{\ln \left(1-e^{-a}\right)}{1-e^{-a}}+\frac{1}{1-e^{-a}} \int_{1}^{\infty} \frac{e^{-a t}}{t} d t
$$

Finally,

$$
M_{3}=-\frac{e^{a}}{b} \int_{0}^{1} e^{-a t}\left(\sum_{j=1}^{\infty}\left(\begin{array}{l}
t \\
j
\end{array}\right) b^{j}\right) \psi(t+1) d t=-\frac{e^{a}}{b} \int_{0}^{1}\left(1-e^{-a t}\right) \psi(t+1) d t .
$$

A direct computation shows that $\int_{0}^{1} \psi(t+1) d t=0$, and integration by parts gives

$$
M_{3}=\frac{a}{1-e^{-a}} \int_{0}^{1} e^{-a t} \ln \Gamma(t+1) d t .
$$

The identity $\ln \Gamma(t+1)=\ln \Gamma(t)+\ln t$ now yields

$$
M_{3}=\frac{a}{1-e^{-a}}\left(\int_{0}^{1} e^{-a t} \ln t d t+\int_{0}^{1} e^{-a t} \ln \Gamma(t) d t\right) .
$$

Replacing (2.17), (2.19) and (2.21) into (2.17) provides an expression for $M(a)$ :

$$
\begin{aligned}
M(a) & =\frac{a}{1-e^{-a}}+\frac{\gamma}{a}+\frac{\ln \left(1-e^{-a}\right)}{1-e^{-a}} \\
& +\frac{a}{1-e^{-a}} \int_{0}^{\infty} e^{-a t} \ln t d t+\frac{a}{1-e^{-a}} \int_{0}^{1} e^{-a t} \ln \Gamma(t) d t .
\end{aligned}
$$

The term $\gamma / a$ comes from the index $j=0$ in the sum (2.18). The main result presented here now follows from (1.1). This settles a conjecture of O. Oloa presented in [13.

Theorem 2.1. If $0<a<\ln 2$, then

$$
M(a)=\frac{\gamma}{a}+\frac{a+\ln \left(1-e^{-a}\right)-\gamma-\ln a}{1-e^{-a}}+\frac{a}{1-e^{-a}} \int_{0}^{1} e^{-a t} \ln \Gamma(t) d t .
$$

The above result is complementary to Theorem 1.1

Corollary 2.1. If $0<a<\ln 2$, then

$$
M(a)=\frac{\gamma}{a}+\frac{a+\ln \left(1-e^{-a}\right)+\Gamma(0, a)}{1-e^{-a}}+\frac{1}{1-e^{-a}} \int_{0}^{1} e^{-a t} \psi(t+1) d t,
$$

where $\Gamma(0, a)$ is the incomplete gamma function.

Proof. Split up the first integral in (2.25) and integrate by parts.

The derivative of (2.1) at $a=0$, the classical values

$$
\int_{0}^{1} \ln \Gamma(t) d t=\frac{1}{2} \ln 2 \pi
$$

and

$$
\int_{0}^{1} t \ln \Gamma(t) d t=\frac{\zeta^{\prime}(2)}{2 \pi^{2}}+\frac{1}{6} \ln 2 \pi-\frac{\gamma}{12}
$$

obtained in [4], give

$$
\int_{0}^{\pi / 2} \frac{x^{2} \ln (2 \cos x) d x}{\left(x^{2}+\ln ^{2}(2 \cos x)\right)^{2}}=\frac{7 \pi}{192}+\frac{\pi \ln 2 \pi}{96}-\frac{\zeta^{\prime}(2)}{16 \pi} .
$$


Further differentiation of (2.1) produces the evaluation of a family of integrals similar to (2.28).

The integral in (2.1) can be expressed in an alternative form. Define

$$
\Lambda(z):=\lim _{n \rightarrow \infty}\left(\sum_{j=1}^{n} \frac{j}{j^{2}+z^{2}}-\ln n\right) .
$$

Observe that $\Lambda(0)=\gamma$, so $\Lambda(z)$ is a generalization of Euler's constant.

Lemma 2.3. Let $a>0, c=1-e^{-a}$ and define $A:=\ln 2 \pi+\gamma$. Then

$$
\int_{0}^{1} e^{-a t} \ln \Gamma(t) d t=\frac{A(a-c)}{a^{2}}-\frac{c}{2 a} \Lambda\left(\frac{a}{2 \pi}\right)+2 c \sum_{j=1}^{\infty} \frac{\ln j}{a^{2}+4 \pi^{2} j^{2}} .
$$

Proof. Expand the exponential into a MacLaurin series and use the value

$$
\begin{aligned}
\int_{0}^{1} t^{n} \ln \Gamma(t) d t & =\frac{1}{n+1} \sum_{k=1}^{\left\lfloor\frac{n+1}{2}\right\rfloor}(-1)^{k}\left(\begin{array}{c}
n+1 \\
2 k-1
\end{array}\right) \frac{(2 k) !}{k(2 \pi)^{2 k}}\left[A \zeta(2 k)-\zeta^{\prime}(2 k)\right] \\
& -\frac{1}{n+1} \sum_{k=1}^{\left\lfloor\frac{n}{2}\right\rfloor}(-1)^{k}\left(\begin{array}{c}
n+1 \\
2 k
\end{array}\right) \frac{(2 k) !}{2(2 \pi)^{2 k}} \zeta(2 k+1)+\frac{\ln \sqrt{2 \pi}}{n+1}
\end{aligned}
$$

given as (6.14) in [4. Then interchange the resulting double sums.

The next corollary follows from the identity $M(a)=L(a)+\gamma / a$.

Corollary 2.2. If $0<a<\ln 2$ and $c=1-e^{-a}$, then

$$
\int_{0}^{\infty} e^{-a t} \ln \Gamma(t) d t=-\frac{\gamma+\ln a}{a c e^{a}}+\frac{A(a-c)}{a^{2} c}-\frac{1}{2 a} \Lambda\left(\frac{a}{2 \pi}\right)+2 \sum_{j=1}^{\infty} \frac{\ln j}{a^{2}+4 \pi^{2} j^{2}}
$$

Lemma 2.4. Let $f(t)=2^{-t} \ln \Gamma(t)$. Then

$$
\begin{aligned}
\int_{0}^{\infty} f(t) d t & =2 \int_{0}^{1} f(t) d t-\frac{\gamma+\ln \ln 2}{\ln 2} \\
\int_{0}^{\infty} t f(t) d t & =2 \int_{0}^{1}(t+1) f(t) d t-\frac{(\gamma+\ln \ln 2)(1+2 \ln 2)-1}{\ln ^{2} 2}
\end{aligned}
$$

Proof. The function $f(t)$ satisfies $f(t+1)=\frac{1}{2} f(t)+\frac{1}{2} 2^{-t} \ln t$. Splitting the integral

$$
\int_{0}^{\infty} f(t) d t=\int_{0}^{1} f(t) d t+\int_{0}^{\infty} f(t+1) d t
$$

and using (1.1) gives the first result. The proof of (2.33) is similar; it only requires differentiating (1.1) with respect to the parameter $a$.

The reader will check that (2.32) is equivalent to the continuity of $M(a)$ at $a=\ln 2$. The identity (2.33) provides a proof of the next theorem, which in itself is worthy of singular (pun intended) interest.

Theorem 2.2. The jump of $M^{\prime}(a)$ at $a=\ln 2$ is 4 . 


\section{Conclusions}

The integral

$$
M(a):=\frac{4}{\pi} \int_{0}^{\pi / 2} \frac{x^{2} d x}{x^{2}+\ln ^{2}\left(2 e^{-a} \cos x\right)}
$$

satisfies

$$
M(a)=\frac{\gamma}{a}+\int_{0}^{\infty} e^{-a t} \psi(t+1) d t
$$

for $a>\ln 2$ and

$$
M(a)=\frac{\gamma}{a}+\frac{a+\ln \left(1-e^{-a}\right)+\Gamma(0, a)}{1-e^{-a}}+\frac{1}{1-e^{-a}} \int_{0}^{1} e^{-a t} \psi(t+1) d t
$$

for $0<a \leq \ln 2$.

\section{REFERENCES}

[1] T. Amdeberhan, L. Medina, and V. Moll. The integrals in Gradshteyn and Ryzhik. Part 5: Some trigonometric integrals. Scientia, 15:47-60, 2007

[2] T. Amdeberhan, V. Moll, J. Rosenberg, A. Straub, and P. Whitworth. The integrals in Gradshteyn and Ryzhik. Part 9: Combinations of logarithms, rational and trigonometric functions. Scientia, to appear.

[3] D. Borwein and J. Borwein. On an intriguing integral and some series related to $\zeta(4)$. Proc. Amer. Math. Soc., 123:1191-1198, 1995. MR1231029 (95e:11137)

[4] O. Espinosa and V. Moll. On some definite integrals involving the Hurwitz zeta function. Part 1. The Ramanujan Journal, 6:159-188, 2002. MR.1908196 (2003f:11127)

[5] L. Euler. Exercitationes analyticae. Novi commentarii academiae scienticarum petropolitanae, 17, 1772, 173-204. In Opera Omnia, volume 15, pages 131-167. Teubner, Berlin, 1924.

[6] M. L. Glasser and D. Manna. On the Laplace transform of the psi-function. "Tapas in Experimental Mathematics" (T. Amdeberhan and V. Moll, eds.), Contemporary Mathematics, vol. 457, Amer. Math. Soc., Providence, RI, 2008, pp. 193-202.

[7] I. S. Gradshteyn and I. M. Ryzhik. Table of Integrals, Series, and Products. Edited by A. Jeffrey and D. Zwillinger. Academic Press, New York, 7th edition, 2007. MR1773820 (2001c:00002)

[8] K. S. Kolbig. On the integral $\int_{0}^{\pi / 2} \log ^{n} \cos x \log ^{p} \sin x d x$. Math. Comp., 40:565-570, 1983. MR689472 (84d:33004)

[9] V. Moll. The integrals in Gradshteyn and Ryzhik. Part 1: A family of logarithmic integrals. Scientia, 14:1-6, 2007. MR2330697

[10] V. Moll. The integrals in Gradshteyn and Ryzhik. Part 2: Elementary logarithmic integrals. Scientia, 14:7-15, 2007. MR2330698

[11] V. Moll. The integrals in Gradshteyn and Ryzhik. Part 3: Combinations of logarithms and exponentials. Scientia, 15:31-36, 2007. MR2367911

[12] V. Moll. The integrals in Gradshteyn and Ryzhik. Part 4: The gamma function. Scientia, 15:37-46, 2007. MR2367912

[13] O. Oloa. Some Euler-type integrals and a new rational series for Euler's constant. Contemporary Mathematics, "Tapas in Experimental Mathematics" (T. Amdeberhan and V. Moll, eds.), Contemporary Mathematics, vol. 457, Amer. Math. Soc., Providence, RI, 2008, pp. 237-248.

[14] M. Petkovsek, H. Wilf, and D. Zeilberger. $A=B$. A K Peters, Ltd., 1st edition, 1996. MR.1379802 (97j:05001) 
[15] I. Vardi. Integrals, an introduction to analytic number theory. Amer. Math. Monthly, 95:308315, 1988. MR935205 (89b:11073)

[16] Z. Yue and K.S. Williams. Values of the Riemann zeta function and integrals involving $\log \left(2 \sinh \frac{\theta}{2}\right)$ and $\log \left(2 \sin \frac{\theta}{2}\right)$. Pac. Jour. Math., 168:271-289, 1995. MR1339953(96f:11170)

Department of Mathematics, Tulane University, New Orleans, Louisiana 70118

E-mail address: tamdeber@tulane.edu

Departmento de Física, Universidad Téc. Federico Santa María, Valparaiso, Chile

E-mail address: olivier.espinosa@usm.cl

Department of Mathematics, Tulane University, New Orleans, Louisiana 70118

E-mail address: vhm@math.tulane.edu 